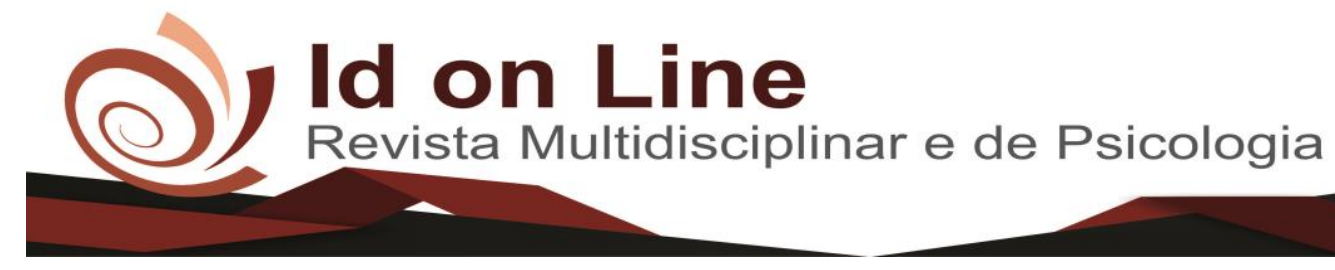

DOI: 10.14295/idonline.v14i53.2876

Artigo

\title{
Tratamentos para a Lipodistrofia Localizada em Fumantes: Revisão de Literatura
}

Valeria Hipólito $^{1}$; Zamia Aline Barros Ferreira ${ }^{2}$

\begin{abstract}
Resumo: Os danos causados pelo consumo de produtos do tabaco, principalmente os de inalação da fumaça do cigarro, é múltipla: doenças cardiovasculares, bronquite crônica e enfisema, vários tipos de câncer etc., além disso, o vício em cigarro pode engordar. Objetivo: analisar os benefícios dos procedimentos estéticos no tratamento da lipodistrofia localizada em fumantes e seus impactos na. Método: Trata-se de uma revisão integrativa de literatura, realizada através das bases de dados BVS (Biblioteca Virtual de Saúde), LILACS (Literatura Latino-Americana em Ciências da Saúde), e Scielo (Biblioteca Eletrônica Científica Online), no período de agosto a outubro de 2020. Resultados: Os estudos apontaram que existem muitos tipos de tratamentos que podem contribuir para reduzir a lipodistrofia em fumantes. Conclusão: Conclui-se que Ultracavitação, carboxiterapia, e radiofrequência, são tratamentos eficazes para a lipodistrofia localizada em fumantes, e seus resultados satisfatórios contribuem sobremaneira para aumento da autoestima de mulheres.
\end{abstract}

Palavras-Chave: Tabagismo. Lipodistrofia. Tratamentos estéticos.

\section{Treatments for Localized Lipodystrophy in Smokers: Literature Review}

\begin{abstract}
The damage caused by the consumption of tobacco products, especially those caused by inhalation of cigarette smoke, is multiple: cardiovascular diseases, chronic bronchitis and emphysema, various types of cancer, etc. In addition, cigarette addiction can make you fat. Objective: to analyze the benefits of esthetic procedures in the treatment of localized lipodystrophy in smokers and their impacts on. Method: It is an integrative literature review, carried out through the databases VHL (Virtual Health Library), LILACS (Latin American Literature in Health Sciences), and Scielo (Electronic Scientific Library Online), in the period of August to October 2020. Results: Studies have shown that there are many types of treatments that can contribute to reducing lipodystrophy in smokers. Conclusion: It is concluded that ultracavitation, carboxitherapy, and radiofrequency, are effective treatments for localized lipodystrophy in smokers, and their satisfactory results contribute greatly to increase the selfesteem of women.
\end{abstract}

Keywords: Smoking. Lipodystrophy. Aesthetic treatments.

\footnotetext{
${ }^{1}$ Discente do curso de Cosmetologia e estética, na Faculdade Independente do Nordeste - FAINOR. Vitória da Conquista, Bahia, Brasil. valeriahipolitob@hotmail.com;

2 Docente na Faculdade Independente do Nordeste - FAINOR. Vitória da Conquista, Bahia, Brasil.
} 


\section{Introdução}

O tabagismo é uma das principais causas de doença e morte prematura no Brasil. Embora o uso de cigarros tenha diminuído lentamente nos Estados Unidos, muitas alternativas ganharam popularidade (MELLO, 2018). O tabagismo e a exposição à fumaça do tabaco causam cerca de 480.000 mortes prematuras a cada ano nos Estados Unidos. Dessas mortes prematuras, cerca de $36 \%$ são de câncer, $39 \%$ são de doenças cardíacas e derrames e $24 \%$ são de doenças pulmonares. A taxa de mortalidade entre os fumantes é quase três vezes maior do que nas pessoas que nunca fumaram (PINTO, 2016).

Fumar compromete quase todos os órgãos do corpo e do sistema de órgãos e diminui a saúde geral de uma pessoa. O tabagismo pode causar diversos tipos de câncer, dentre eles o de pulmão (VAN, 2015). Assim sendo este também pode causar doenças cardíacas e respiratórias, acidente vascular cerebral, aneurismas doenças pulmonares, diabetes, osteoporose, artrite reumatoide. Além disso, o tabagismo causa inflamação e enfraquece a função imune (PNEUMOIMAGEM, 2016). Tornando este um problema de saúde pública.

O vício em cigarro pode engordar, especialmente um grupo em que os quilos adicionais são ainda mais desastrosos: as mulheres obesas. De acordo com um estudo realizado pela Universidade de Washington, o cigarro dificulta a percepção do sabor de alimentos açucarados e gordurosos, comprometendo a sensação de saciedade por esses produtos (BRAY, 2015).

Nesse contexto, depósitos de gordura localizados são acúmulos de células adiposas ou excesso de gordura localizada em certas áreas do corpo, determinados por herança genética, hormônios, sexo etc., caracterizados por serem resistentes à dieta e à prática habitual de exercício físico. Uma das áreas do corpo onde elas aparecem com mais frequência em mulheres e homens é a região do abdômen e da cintura (SZKLO, 2015).

A eliminação da gordura corporal e a eliminação da celulite são um problema comum no campo da estética, mas nem todos os tratamentos e tecnologias são iguais. Técnicas minimamente invasivas para eliminar adiposidades localizadas requerem efetivamente uma abordagem abrangente por um profissional médico para obter resultados de maneira segura, eficaz e duradoura (OLIVEIRA, 2016). Tratamentos como cavitação, a combinação de radiofrequência e carboxiterapia e eletroestimulação conseguem diminuir e eliminar a gordura localizada no abdômen (SILVA, 2015).

O tabagismo é atualmente um problema de saúde pública em todo o mundo, além de ser um fator de risco modificável para muitas comorbidades, inclusive doença cardiovascular. A 
relação entre o tabagismo e o estado nutricional tem sido muito estuda. A obesidade, particularmente a obesidade central, é um grande fator de risco para doenças cardiovasculares, afetando principalmente adultos e idosos.

A gordura abdominal é um indicador de gordura visceral, que causa complicações metabólicas e aumentando o risco de morte (NANDA, 2015). A prevalência do tabagismo permanece alta e a obesidade está aumentando na população, contribuindo para um aumento significativo da incidência de doença cardiovascular (OUCHI, 2017).

Nesse contexto, o objetivo desta pesquisa é analisar como os procedimentos estéticos no tratamento da lipodistrofia localizada em fumantes e seus impactos na autoestimaanalisar a associação entre tabagismo e obesidade abdominal em mulheres, bem como avaliar como os procedimentos estéticos podem auxiliar na diminuição da gordura abdominal de mulheres fumantes e na elevação da sua autoestima.

\section{Materiais e Métodos}

Trata-se de uma revisão integrativa de literatura, visto que apresenta uma abordagem mais sistemática e rigorosa do que a revisão narrativa da literatura. Esse tipo de revisão tem como foco fundamental sintetizar conhecimentos sobre metodologia, conhecimentos teóricos ou sobre a pesquisa realizada, delineando uma conclusão sobre um tema específico (GALVÃO, 2003).

Nesse tipo de revisão, a síntese do conhecimento inclui uma abordagem ampla do campo de estudo que pode incluir tanto a literatura empírica quanto a teórica para atingir os objetivos da revisão (WHITTEMORE, 2005). Nesse caso, pesquisa é entendida como primária (pesquisa clínica) e secundária (revisões sistemáticas), incluindo artigos conceituais ou teóricos.

Esse tipo de revisão, também chamada de revisão crítica, visa demonstrar que o autor pesquisou extensivamente a literatura e avaliou criticamente sua qualidade. Vai além da mera descrição dos artigos identificados e inclui um certo grau de análise e inovação conceitual.

A pergunta norteadora estabelecida foi: Quais os benefícios dos tratamentos estéticos para portadores de lipodistrofia localizada em fumantes? A coleta de dados, ocorreu no período de agosto a outubro de 2020. Os descritores utilizados na pesquisa foram os seguintes: Tabagismo. Lipodistrofia. Tratamentos estéticos. Os critérios de inclusão adotados foram: artigos que abordam a temática no idioma em língua portuguesa, inglesa e espanhol, publicados nos anos de 2009 a 2018; publicações completas com resumos disponíveis e indexados nas 
bases de dados: BVS (Biblioteca Virtual de Saúde), LILACS (Literatura Latino-Americana em Ciências da Saúde) e Scielo (Biblioteca Eletrônica Científica Online). Foram excluídos os artigos dos anos que excedem essas datas e em outros idiomas.

Para selecionar as evidências encontradas nas investigações, buscou-se realizar uma classificação dividida da seguinte forma: evidências s de pelo menos um ensaio clínico controlado bem delineado; Pesquisa com método bem desenhado de ensaios clínicos não randomizados; Evidências de estudos de coorte e caso-controle bem planejados; Revisões sistemáticas de estudos descritivos e qualitativos; Evidências de um único estudo descritivo ou qualitativo e opiniões de autoridades especialistas na área estudada

\section{Resultados}

Para obtenção dos resultados desse estudo, a princípio foram encontrados 23 artigos científicos que tratavam sobre o assunto com descritores, Tabagismo. Lipodistrofia. Procedimentos e estética, após a leitura, foram filtrados, de acordo com os critérios de inclusão, 15 artigos, que passaram para a segunda etapa. Na segunda etapa, após a leitura dos resumos e análise na íntegra verificando a relevância ao tema, permaneceram 07 artigos? que serviram de base para esta pesquisa. Compuseram a amostra dos resultados desta pesquisa cinco artigos científicos, conforme apresentado no Quadro 1, que está organizado da seguinte forma: Autor, ano de publicação, objetivos e principais resultados.

Quadro 1 - Artigos selecionados segundo ano de publicação, auto e ano de publicação, título, objetivos e principais resultados. Vitória da Conquista, Bahia, Brasil. 2020.

\begin{tabular}{|c|c|c|c|}
\hline $\begin{array}{c}\text { AUTOR/ } \\
\text { ANO }\end{array}$ & TÍTULO & OBJETIVOS & PRINCIPAIS RESULTADOS \\
\hline $\begin{array}{l}\text { Scorza; } \\
\text { Borges, } \\
2009 .\end{array}$ & $\begin{array}{l}\text { Carboxiterapia: } \\
\text { uma revisão } \\
\text { integrativa } \\
\text { literatura }\end{array}$ & $\begin{array}{l}\text { Esclarecer aos } \\
\text { profissionais das } \\
\text { áreas médica e } \\
\text { fisioterapêutica } \\
\text { mais uma fonte } \\
\text { de pesquisa, haja } \\
\text { vista a escassez } \\
\text { de artigos que } \\
\text { relatam o } \\
\text { aludido assunto }\end{array}$ & $\begin{array}{l}\text { Trata-se de um método seguro que } \\
\text { exige equipamento especializado, vem } \\
\text { mostrando resultados significativos e } \\
\text { tem seu uso respaldado pela literatura } \\
\text { científica. }\end{array}$ \\
\hline $\begin{array}{l}\text { Lima. et.al. } \\
2015\end{array}$ & $\begin{array}{l}\text { Eletrolipólise e } \\
\text { Carboxiterapia para } \\
\text { redução de gordura } \\
\text { localizada }\end{array}$ & $\begin{array}{l}\text { Verificar os } \\
\text { benefícios da } \\
\text { Eletrolipólise e } \\
\text { Carboxiterapia }\end{array}$ & $\begin{array}{l}\text { Os tratamentos apresentados são } \\
\text { invasivos, realizados apenas por } \\
\text { profissionais capacitados }\end{array}$ \\
\hline
\end{tabular}

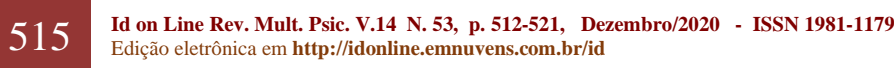




\begin{tabular}{|c|c|c|c|}
\hline & & $\begin{array}{l}\text { na redução de } \\
\text { gordura } \\
\text { localizada }\end{array}$ & \\
\hline $\begin{array}{l}\text { Szklo; et.al, } \\
2015\end{array}$ & $\begin{array}{l}\text { Fumantes no Brasil: } \\
\text { quem são eles? }\end{array}$ & & $\begin{array}{l}\text { Para ambos os sexos, à medida que a } \\
\text { prevalência do tabagismo diminuiu no } \\
\text { Brasil, aumentou a proporção de } \\
\text { fumantes que pararam de fumar. }\end{array}$ \\
\hline $\begin{array}{l}\text { Silva; et.al, } \\
2016\end{array}$ & $\begin{array}{l}\text { Oferecer, } \\
\text { particularmente aos } \\
\text { pneumologistas, } \\
\text { informações sobre } \\
\text { o tabagismo que } \\
\text { auxiliem na sua } \\
\text { prática diária de } \\
\text { atendimento aos } \\
\text { pacientes fumantes } \\
\text { no consultório }\end{array}$ & $\begin{array}{l}\text { Verificar As } \\
\text { políticas de } \\
\text { controle e os } \\
\text { recursos } \\
\text { terapêuticos para } \\
\text { o tabagismo }\end{array}$ & $\begin{array}{l}\text { É preciso atuar com maior ímpeto em } \\
\text { prol das políticas de saúde e das } \\
\text { normas de convívio social que } \\
\text { contribuem diretamente para melhorar } \\
\text { a saúde e a vida. Nesse aspecto, os } \\
\text { pneumologistas podem ter um papel de } \\
\text { maior destaque na medida em que se } \\
\text { envolvam com o tratamento dos } \\
\text { fumantes, a aplicação da lei antifumo e } \\
\text { as políticas de saúde relacionadas às } \\
\text { doenças respiratórias. }\end{array}$ \\
\hline $\begin{array}{l}\text { REIS, } \\
\text { Carolina } \\
\text { Mattei de, } \\
2018\end{array}$ & \begin{tabular}{ll}
\multicolumn{2}{|l}{ Avaliação temporal } \\
dos efeitos & da \\
carboxiterapia & no \\
tratamento & da \\
lipodistrofia & \\
localizada &
\end{tabular} & $\begin{array}{l}\text { Identificar os } \\
\text { efeitos } \\
\text { temporais do uso } \\
\text { da } \\
\text { carboxiterapia } \\
\text { na lipodistrofia } \\
\text { localizada }\end{array}$ & $\begin{array}{l}\text { Ocorreu diminuição dos níveis de } \\
\text { glicose. Não houve diferença } \\
\text { estatística de perimetria, adipometria. }\end{array}$ \\
\hline $\begin{array}{l}\text { Silva, et.al. } \\
2018\end{array}$ & $\begin{array}{l}\text { Ultracavitação para } \\
\text { gordura localizada } \\
-\quad \text { revisão de } \\
\text { literatura }\end{array}$ & $\begin{array}{l}\text { Analisar a } \\
\text { eficácia da } \\
\text { Ultracavitação } \\
\text { para a redução } \\
\text { de medidas } \\
\text { localizadas }\end{array}$ & $\begin{array}{l}\text { O procedimento é agregado com } \\
\text { práticas físicas e uma boa alimentação } \\
\text { para que se tenha resultados } \\
\text { proveitosos }\end{array}$ \\
\hline $\begin{array}{l}\text { Bastos; } \\
\text { Nogueira, } \\
2020\end{array}$ & $\begin{array}{ll}\text { Analisar } & \text { os } \\
\text { benefícios } & \text { da } \\
\text { técnica } & \text { da } \\
\text { carboxiterapia } & \text { no } \\
\text { tratamento } & \text { da } \\
\text { adiposidade } & \\
\text { abdominal } & \\
\end{array}$ & $\begin{array}{l}\text { Analisar os } \\
\text { benefícios da } \\
\text { técnica da } \\
\text { carboxiterapia } \\
\text { no tratamento da } \\
\text { adiposidade } \\
\text { abdominal }\end{array}$ & $\begin{array}{l}\text { A carboxiterapia oferece inúmeros } \\
\text { benefícios no tratamento da } \\
\text { adiposidade abdominal e está } \\
\text { associada a um baixo risco de } \\
\text { complicações e não limita todos os } \\
\text { dias atividade profissional }\end{array}$ \\
\hline
\end{tabular}

Fonte: Dados da pesquisa, 2020

\section{Discussão}

Os resultados dos estudos analisados nesta pesquisa demonstraram que os procedimentos estéticos são eficazes no tratamento da lipodistrofia localizada em fumantes e 
impactam de forma positiva na autoestima de mulheres fumantes, pois reduzem a lipodistrofia causada pelo consumo do tabagismo.

Lipodistrofia é o acúmulo de gordura em certas áreas do corpo que desarmoniza a figura. É produzido por uma maior concentração de células adiposas por unidade de volume nessas áreas, em comparação com o resto do corpo. O aparecimento da lipodistrofia responde a influências hereditárias e hormonais. Esse fato significa que em cada sexo, eles têm um local típico e diferente (CAR, 2003).

Em um estudo realizado sobre os efeitos da carboxiterapia no tratamento da lipodistrofia, Reis (2018) encontraram como resultado redução nos níveis de perimetria e adipometria.

Corrobando com Borges e Scorza (2016), a infusão do gás no plano hipodérmico é capaz de promover vasodilatação, aumento do fluxo sanguíneo, hiperoxigenação tecidual e estímulo a lipólise. Ao ser aplicado no tecido subcutâneo ocorre o estímulo circulatório. O trauma provocado pela agulha e a infusão do gás geram hiperemia local e um processo inflamatório é instaurado, este possui como intuito a cicatrização e o reparo tecidual, aumento do número de vasos sanguíneos, fibroblastos e do calibre vascular, além da melhora do fluxo sanguíneo.

O estudo de Silva; et.al. (2016), corroborando com o estudo de Szklo (2015), demostrou que os danos causados pelo consumo de produtos do tabaco, principalmente os de inalação da fumaça do cigarro, é múltipla: doenças cardiovasculares, bronquite crônica e enfisema, vários tipos de câncer etc. $\mathrm{O}$ carbono é uma das substâncias tóxicas que causam danos cardiovasculares. Contudo, o maior efeito prejudicial do tabaco é exercido por meio de ações procoagulantes. A influência do uso de tabaco sobre a gordura abdominal ainda não é clara em muitos estudos estudo, a análise de regressão linear multivariada confirma a associação entre indicadores antropométricos de adiposidade abdominal e tabagismo após o ajuste para alguns fatores, inclusive consumo de álcool

O estudo de Lima et.al (2015) demostrou que existem vários recursos eletroterápicos e cosméticos utilizados para o tratamento da lipodistrofia, destacando a carboxiterapia é uma terapia que se utiliza do gás carbônico medicinal administrado por via subcutânea, promovendo assim alguns efeitos fisiológicos, tais como melhora a da circulação e oxigenação tecidual.

A carboxiterapia não possui muitas contraindicações e os efeitos secundários da carboxiterapia são: dor na região onde houve a aplicação, hematomas e equimoses (pequenos e somem em cerca de 30 minutos) e o aumento da temperatura local. Como resultado percebe-se o aumento do fluxo sanguíneo influenciado pelo hipercapnia local e como a hemoglobina possui 
grande afinidade pelo $\mathrm{CO} 2$, há também uma maior disponibilidade de oxigênio tissular e melhoria na microcirculação local sem ser tóxico (REIS, 2018).

No entanto o estudo de Bastos e Nogueira (2020), evidenciou que a carboxiterapia é contraindicada para mulheres grávidas; pessoas com insuficiência cardíaca ou insuficiência respiratória, insuficiência renal e hepática, diabéticos, pacientes com problemas psicológicos; epiléticos; hipertensivos e portadores de doenças crônicas a exemplo do lúpus eritematoso sistêmico. Esse tratamento é contraindicado em indivíduos epileticos, insuficiência cardiorrespiratória, insuficiência renal, hipertensão arterial severa, gestantes e pessoas com alterações psíquicas.

Os tratamentos estéticos mais realizados para gordura localizada são: eletroporação, que consiste na emissão de ondas eletromagnéticas pulsadas, atérmicas e não ionizáveis (com predominância do campo elétrico), a fim de produzir uma alteração no potencial da membrana. A Ultracavitação: que são baseados na baixa frequência do ultrassom. Os ultrassons criam bolhas nos líquidos, que crescem gradualmente até atingirem um tamanho crítico em que se quebram e o conteúdo celular é liberado. Ácidos graxos livres, insolúveis em água, são transportados para o fígado e processados como qualquer ácido gordurosos da dieta e são eliminados pela urina (LIMA, et, al 2015).

A radiofrequência é um dos equipamentos médico-estéticos mais utilizados para o manejo de diferentes condições cutâneas na área da Medicina Estética. Essa não altera a epiderme produz um aquecimento dérmico com estimulação molecular, gerando um estímulo regenerativo e reparador. As tecnologias mais utilizadas são as radiofrequências monopolares (quando o ânodo está a uma distância do cátodo de maior profundidade), bipolar (quando o ânodo está próximo a um cátodo de menor profundidade) e tripolar (quando existem três polos e diferentes profundidades de radiofrequência) (SILVA, 2018).

Segundo Silva (2018), o Ultrassom é um tratamento estético não invasivo: sua ação nos tecidos provoca uma micromassagem celular e molecular, alcançando efeitos terapêuticos. Essa técnica induz a vasodilatação e ajuda a melhorar o metabolismo do tecido adiposo, restabelecendo a microcirculação afetada pela celulite. Este tratamento apresenta excelentes efeitos anti-edematosos, aumentando a drenagem linfática e a permeabilidade da pele, além de ter um importante efeito trófico e anti-inflamatório. É muito útil como complemento para outros tratamentos estéticos não invasivos. É específico para o abdômen, quadril, parte interna das coxas, braços e costas. No estudo do autor esse recurso foi utilizado no tratamento da 
adiposidade localizada na área abdominal, aplicando um gel condutor e, em seguida, trazendo a cabeça do equipamento diretamente para a pele.

A ultracavitação é uma técnica não invasiva usada na medicina estética, baseada no ultrassom que destrói adipócitos ou células adiposas, ao usar uma frequência de vibração específica a uma determinada potência, a moléculas de uma estrutura específica, gerando microbolhas que explodem ou implodem, quebrando essa estrutura seletivamente e não outra. No caso das células adiposas, observou-se que a frequência de ressonância é alcançada entre 37 e $42 \mathrm{Khz}$. A implosão favorece a decomposição das gorduras e estas são liberadas, em cadeias mais curtas, na forma de ácidos graxos e glicerol, para sua posterior eliminação através do sistema linfático, urina e fezes (SILVA, 2018).

Sendo assim, de forma geral todos esses procedimentos podem ser utilizados para o tratamento da Lipodistrofia em fumantes, cabendo ao profissional uma avaliação ou anamnese adequada para cada caso e observar se existe alguma contraindicação.

\section{Considerações Finais}

Esse estudo teve como objetivo analisar como os procedimentos estéticos no tratamento da lipodistrofia localizada em fumantes e seus impactos na autoestima Os achados desse estudo, evidenciaram que existe uma grande variedade de tratamentos estéticos, que produzem resultados eficientes na lipodistrofia localizada de forma geral, bem como podemos dizer que esses tratamentos também tornam-se eficazes em fumantes.

Ocorreram dificuldades para encontrar estudos que tratassem da temática abordada, haja vista que há uma escassez de artigos que associassem os efeitos dos tratamentos estéticos na lipodistrofia de forma mais específica em fumantes. A realização dessa pesquisa propiciou, ainda, reflexões e questionamentos, com desenvolvimento de senso crítico, dessa temática que indiretamente envolve toda sociedade

Espera-se com este estudo contribuir para que os profissionais reflitam sobre a importância da realização desses procedimento, e que haja equipes multidisciplinares que oriente os pacientes fumantes a buscarem soluções estéticas para reduzir a gordura localizada, ressalta ainda a importância da compreensão desse fenômeno para os profissionais de estética de maneira a subsidiar a formação. 


\section{Referências}

BASTOS; Gabriela Ribeiro; Nogueira, Ana Paula silva. Os Benefícios da Carboxiterapia no Tratamento da Adiposidade Abdominal: Uma Revisão Integrativa. Id on Line Rev. Mult. Psic. V.14, N. 51 p. 157-167, Julho/2020 . Disponível em: Edição eletrônica em http://idonline.emnuvens.com.br/id. Acesso em 30 de novembro de 2020.

BRAY F, FERLAY J, LAVERSANNE M, BREWSTER DH, GOMBE MBALAWA C, KOHLER B, et al. Cancer incidence in five continents: inclusion criteria, highlights from volume $X$ and global status of cancer registration. Int J Cancer.; vol. 137 n.9 p. 2060-71, 2015 Disponível em : https://doi.org/10.1002/ijc.29670 https://doi.org/10.1002/ijc.29670. Acesso em 25 de março de 2020.

Galvão CM, Sawada NO, Mendes IA. A busca das melhores evidências. Rev Esc Enferm USP. Dez; v.37 n.4, p43-50, 2003.

Galvão CM. A prática baseada em evidências: uma contribuição para a melhoria da assistência de enfermagem perioperatória [livre-docência]. Ribeirão Preto (SP): Universidade de São Paulo; 2002.

LIMA; et.al. A eficácia da eletrolipólise na gordura localizada na região abdominal em diferentes frequências. Unicor. Universidade Vale do Rio Verde, 2015. Disponível em: file:///D:/Users/sil/Downloads/Feliciana\%20Saray\%20Costa\%20Veiga,\%20Juciara\%20Caroli ne\%20Leite_A\%20Eficacia\%20da\%20Eletrolipolise \%20na\%20Gordura\%20Localizada\%20n a\%20Regiao\%20Abdominal\%20em\%20Di.pdf. Aceso em 15 de outubro de 2020.

MELO, Robesita Travassos; MARTINS, Albert Eduardo Silva. Importância da dosagem de enzimas cardíacas no diagnóstico de infarto agudo do miocárdio. Repositório institucional Tiradentes.Pernambuco.2018.

NANDA, Diagnósticos de Enfermagem. Definições e classificação 2015-2017/[NANDA Internacional]. Porto Alegre: Artmed, 2015.

OLIVEIRA CASTRO, Yana Thalita Barros et al. Conhecimento e significado do cateterismo cardíaco para pacientes cardiopatas. Revista da Rede de Enfermagem do Nordeste, v. 17, n. 1, p. 29-35, 2016.

OUCHI, Janaína Daniel etal. Tempo de chegada do paciente infartado na unidade de terapia intensiva: a importância do rápido atendimento. Ensaios científicos. Cienc., Biol. Agrar. Saúde, vol. 21, nº 2. P 92-97, 2017.

PINTO MT, Pichon-Riviere A, Biz A, Schluckbier L, Araújo AJ. Relatório Final: Carga das doenças tabaco-relacionadas para o Brasil. São Paulo: Aliança de Controle do Tabagismo; 2016. Disponível

em: http://actbr.org.br/uploads/conteudo/721_Relatorio_Carga_do_tabagismo_Brasil.pdf. Acesso em 05 de abrilde 2020.

PNEUMOIMAGEM São Paulo: Pneumoimagem [updated 2016 Jul 27; Carta de Gramado: Manifesto sobre o Controle do Tabagismo; Available disponível 
emn http://www.pneumoimagem.com.br/noticia_destaque.asp?idnot=175 Acesso em 25 de março de 2020.

REIS, C.M. Avaliação temporal dos efeitos da carboxiterapia no tratamento da lipodistrofia localizada. UNIVATES, 2018. Disponível em: https://www.univates.br/bdu/bitstream/10737/2635/1/2018CarolinaMReis.pdf. Acesso em 28 de outubro de 2020.

RMV, MEYER PF, SANTOS BR, FÉLIX JLO, RONZIO AO. Efectos Del SILVA ultrasonido de alta potencia em La adiposidad localizada. Fisio. vol.37 n.2 p55-59, 2015.

SILVA, Luiz Carlos Corrêa da and COMISSAO DE TABAGISMO DA SBPT et al. Controle do tabagismo: desafios e conquistas. J. bras. pneumol. . 2016, vol.42, n.4, pp.290-298. ISSN 1806-3756. https://doi.org/10.1590/s1806-37562016000000145.

$\mathrm{S}$

ILVA,J.P . SOUZA,F.S . ASSIS,I.B. .PEREIRA,L.P2Ultracavitação para gordura localizada revisão de literatura. Revista Saúde em Foco - Edição no 10 - Ano: 2018. Disponível em: https://portal.unisepe.com.br/unifia/wp-

content/uploads/sites/10001/2018/08/081_ULTRACAVITA\%C3\%87\%C3\%83O-PARA-

GORDURA-LOCALIZADA-_-REVIS\%C3\%830-DE-LITERATURA.pdf. Acesso em 30 de novembro de 2020.

SZKLO A, SOUZA MC, SZKLO M, et al. Smokers in Brazil: who are they? Tob Control Published. DOI:10.1136/tobaccocontrol-2015-052324.

VAN BEEK EJ, MIRSADRAEE S, MURCHISON JT. Lung cancer screening: Computed tomography or chest radiographs? World J Radiol. v.7 n.8, p189-93, 2015. . Disponível em:http://dx.doi.org/10.4329/wjr.v7.i8.189. Aceso em 03 de março de 2020.

Whittemore R, Knafl K. The integrative review: updated methodology. J Adv Nurs. 2005 Dec; 52(5):546-53.

\section{Como citar este artigo (Formato ABNT):}

HIPÓLITO, Valéria; FERREIRA, Zamia Aline Barros. Tratamentos para a Lipodistrofia Localizada em Fumantes: Revisão de Literatura. Id on Line Rev.Mult. Psic., Dezembro/2020, vol.14, n.53, p. 512-521. ISSN: 1981-1179.

Recebido: 02/12/2020;

Aceito: $14 / 12 / 2020$. 